\title{
Association of Anticholinergic Burden with Cognitive and Functional Status in a Cohort of Hospitalized Elderly: Comparison of the Anticholinergic Cognitive Burden Scale and Anticholinergic Risk Scale
}

\author{
Results from the REPOSI Study
}

\author{
Luca Pasina $\cdot$ Codjo D. Djade - Ugo Lucca - Alessandro Nobili • Mauro Tettamanti • \\ Carlotta Franchi $\cdot$ Francesco Salerno $\cdot$ Salvatore Corrao $\cdot$ Alessandra Marengoni \\ Alfonso Iorio · Maura Marcucci · Francesco Violi · Pier Mannuccio Mannucci
}

Published online: 14 December 2012

(C) Springer International Publishing Switzerland 2012

\begin{abstract}
Background Drugs with anticholinergic effects are associated with adverse events such as delirium and falls as well as cognitive decline and loss of independence.

Objective The aim of the study was to evaluate the association between anticholinergic burden and both cognitive and functional status, according to the hypothesis that the cumulative anticholinergic burden, as measured by the Anticholinergic Cognitive Burden (ACB) Scale and Anticholinergic Risk Scale (ARS), increases the risk of cognitive decline and impairs activities of daily living.
\end{abstract}

On behalf of REPOSI Investigators.

Electronic supplementary material The online version of this article (doi:10.1007/s40266-012-0044-x) contains supplementary material, which is available to authorized users.

L. Pasina $(\bowtie)$

Laboratory for Quality Assessment of Geriatric Therapies and Services, Drug Information Service for the Elderly,

Mario Negri Institute for Pharmacological Research,

Via Giuseppe La Masa, 19, 20156 Milan, Italy

e-mail: luca.pasina@marionegri.it

C. D. Djade · U. Lucca · A. Nobili · M. Tettamanti - C. Franchi

Mario Negri Institute for Pharmacological Research, Milan, Italy

F. Salerno

Internal Medicine I, Policlinico IRCCS San Donato,

University of Milan, Milan, Italy

S. Corrao

Dipartimento Biomedico di Medicina Interna e Specialistica,

University of Palermo, Palermo, Italy
Methods This cross-sectional, prospective study (3-month telephone follow-up) was conducted in 66 Italian internal medicine and geriatric wards participating in the Registry of Polytherapies SIMI (Società Italiana di Medicina Interna) (REPOSI) study during 2010. The sample included 1,380 inpatients aged 65 years or older. Cognitive status was rated with the Short Blessed Test (SBT) and physical function with the Barthel Index. Each patient's anticholinergic burden was evaluated using the ACB and ARS scores.

Results The mean SBT score for patients treated with anticholinergic drugs was higher than that for patients receiving no anticholinergic medications as also indicated by the ACB scale, even after adjustment for age, sex, education, stroke and transient ischaemic attack [9.2 (95\% CI 8.6-9.9) vs. 8.5 (95\% CI 7.8-9.2); $p=0.05$ ]. There was a dose-response relationship between total ACB score and cognitive impairment. Patients identified by the ARS

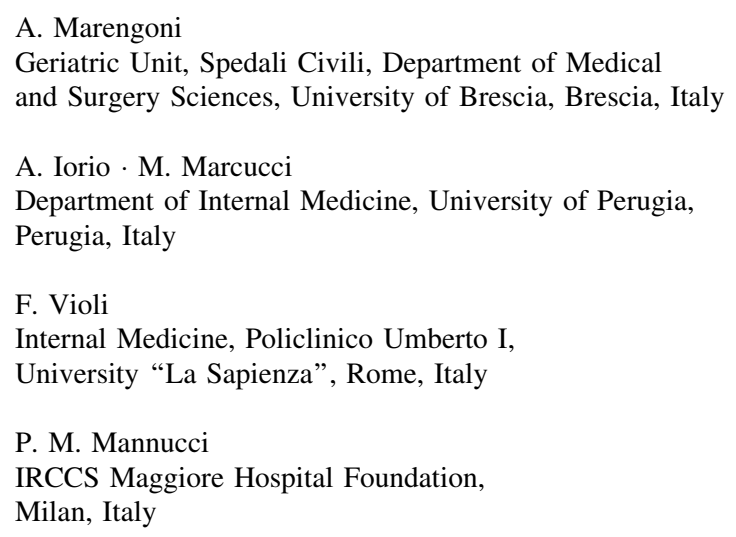


had more severe cognitive and physical impairment than patients identified by the ACB scale, and the dose-response relationship between this score and ability to perform activities of daily living was clear. No correlation was found with length of hospital stay.

Conclusions Drugs with anticholinergic properties identified by the ACB scale and ARS are associated with worse cognitive and functional performance in elderly patients. The ACB scale might permit a rapid identification of drugs potentially associated with cognitive impairment in a doseresponse pattern, but the ARS is better at rating activities of daily living.

\section{Introduction}

Polypharmacy is very common among older adults and may often be needed to improve symptoms, disease-related problems and quality of life [1-3]. However, it may also be a major risk for inappropriate prescribing, poor adherence to therapies, adverse drug events and other adverse health outcomes [4-8]. Elderly patients are at particularly high risk because of multiple illnesses, and there is ample evidence that drugs with anticholinergic properties are especially likely to cause adverse events [9-21] leading to cognitive impairment, delirium, falls and loss of independence [22, 23]. Amongst the oldest subjects, the use of anticholinergic drugs has also been associated with impaired physical performance and functional status [24]. Anticholinergic drugs may affect the brain by blocking the neurotransmitter acetylcholine, and older people are more susceptible to these effects because of several age-related changes, such as a decrease in cholinergic neurons or receptors in the brain, a reduction in hepatic and renal clearance of drugs, and an increase in blood-brain barrier permeability, particularly during an acute physical illness [10, 25-27]. Reducing the use of anticholinergic medications is one predictable way of modifying the risk of this morbidity in elderly people [28].

The Anticholinergic Cognitive Burden (ACB) Scale [29] and the Anticholinergic Risk Scale (ARS) [28] are two scoring systems that rank anticholinergic medications and were developed to predict the risk of anticholinergic adverse effects in older patients. We examined data from the Registry of Polytherapies SIMI (Società Italiana di Medicina Interna) (REPOSI) study, which involved a network of internal medicine and geriatric wards created to collect information on elderly inpatients with multiple diseases and undergoing various therapies (see Appendix S1 for information on the members of the REPOSI Study [Online Resource 1]). Our aim was to assess the association between anticholinergic burden and both cognitive and functional status, according to the hypothesis that the cumulative anticholinergic burden increases the risk of cognitive decline and impairs activities of daily living in elderly patients admitted to internal medicine and geriatric wards.

\section{Methods}

\subsection{Data Collection}

The REPOSI study is a collaborative, independent, voluntary effort by the Italian Society of Internal Medicine (SIMI) and the Mario Negri Institute for Pharmacological Research, and has been described elsewhere in detail [30-35]. It was set up in 2008 to create a network of internal medicine and geriatric wards in order to collect national information on hospitalized elderly patients with multiple diseases often receiving polytherapy. Participation was voluntary and all patients provided signed informed consent. Data collection complied fully with Italian law on personal data protection. Under the applicable legal principles on patients' registries, the study did not require ethical committee approval. The attending physicians completed a standardized web-based Case Report Form, which included information on the following: diagnosis at hospital admission; sociodemographic details; drug treatments at hospital admission, in-hospital stay and discharge; some laboratory parameters; comorbidity according to the Cumulative Illness Rating Scale (CIRS) [36]; performance in basic activities of daily living according to the Barthel Index (BI) [37]; cognitive impairment according to the Short Blessed Test (SBT) [38]; presence of depression according to the Geriatric Depression Scale [39]; clinical events during hospitalization; and outcomes. Patients were followed up 3 months after discharge, and data were collected on any new diagnoses, new hospital admission, drug regimens, adverse drug events and ability to perform activities of daily living (according to the BI).

\subsection{Exposure to Anticholinergic Drugs}

Several drugs have anticholinergic activity, and different scales have been proposed to classify medications according to their anticholinergic effects [28, 29, 40]. The two most recent scales assessing the risk of anticholinergic effects in a clinical setting differ in the number of drugs included, and some drugs are classified differently. The ACB scale (Table 1) [29] is as a practical tool to identify the severity of any anticholinergic negative effect on cognition. Drugs with possible anticholinergic effects are defined as those with serum anticholinergic activity or in vitro affinity for muscarinic receptors but no known clinically relevant cognitive effects (ACB score 1). Drugs with established, clinically relevant cognitive effects are considered definitely anticholinergic (ACB score 2 or 3 ).

The second scale, the ARS (Table 1) [28], estimates the extent to which an individual patient may be at risk of 
Table 1 Anticholinergic Cognitive Burden (ACB) Scale [29] and Anticholinergic Risk Scale (ARS) [28] scores

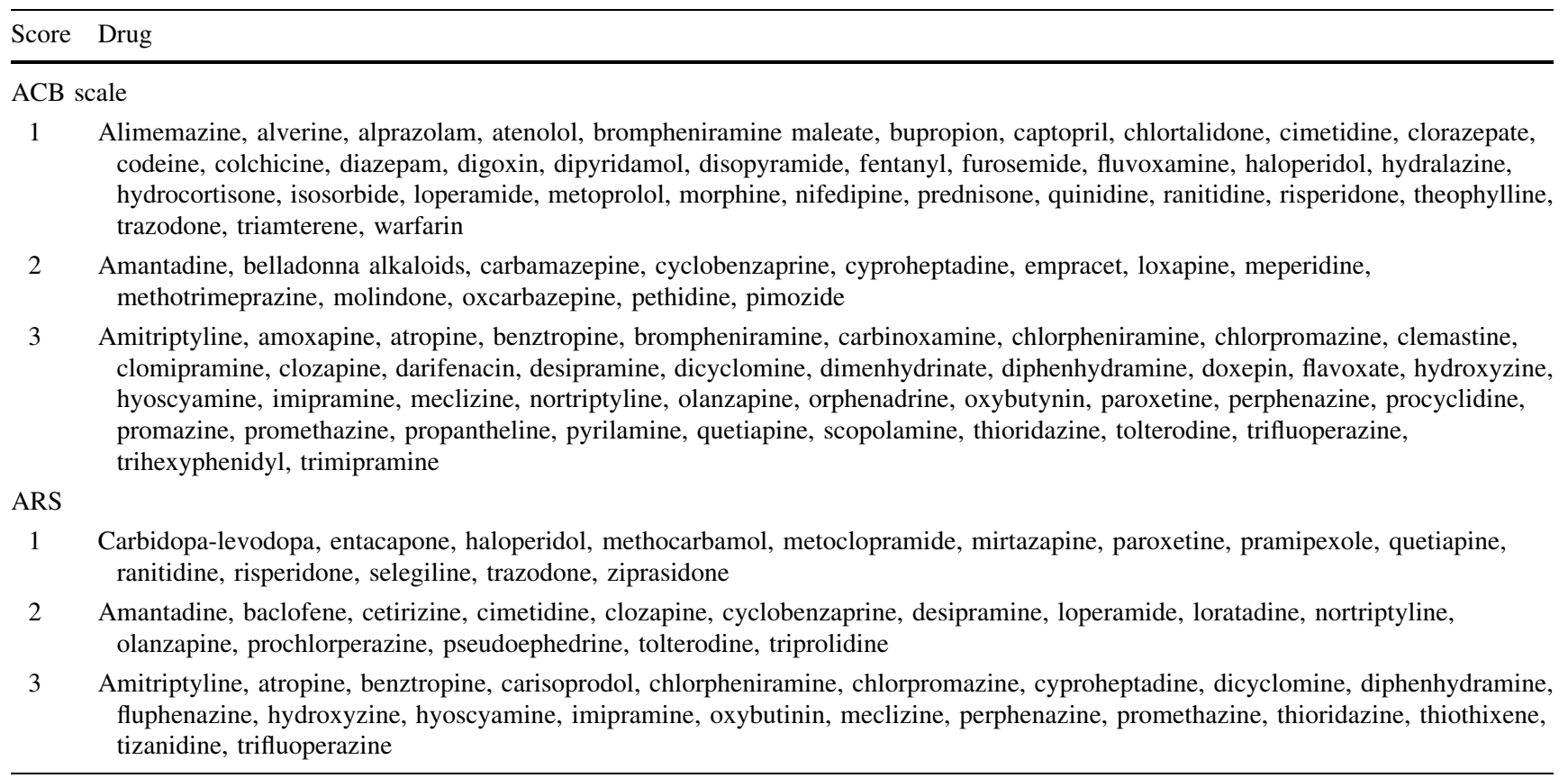

anticholinergic adverse effects that can lead to cognitive dysfunction, delirium and peripheral anticholinergic adverse effects such as dry mouth, dry eyes and constipation. In the ARS, medications are divided according to their anticholinergic potential on a 3-point scale: drugs with moderate anticholinergic potential (ARS score 1), strong potential (ARS score 2) and very strong potential (ARS score 3).

For the purposes of this study, we calculated the drugrelated anticholinergic burden for each patient, using the sum of the points for each anticholinergic medication dispensed at hospital admission, according to both scales.

\subsection{Cognitive Impairment}

The SBT was conducted within 2 days of hospital admission. In cases where patients were considered unstable (such as in those with delirium), the SBT was conducted after stabilization. This is a six-item orientation memory concentration test validated as a measure of cognitive impairment that can distinguish mild, moderate and severe cognitive deficits, with the following cut points: normal cognition (scores of 0-4), questionable impairment (scores of 5-9) and impairment consistent with dementia (scores of 10-28) [41]. To avoid ceiling effects in the SBT scale, we excluded patients with a score of $\geq 25$, with the aim of excluding any patients with a diagnosis of dementia, in which the cognitive effects of anticholinergic medication might be reduced and hard to detect. Among patients with SBT scores of 0-24, we excluded 13 because they had a diagnosis of dementia or were being treated with anticholinesterase agents or memantine.

\subsection{Ability in Basic Activities of Daily Living}

The BI is used to assess ability in basic activities of daily living. It was performance based and was done within 2 days of hospital admission or when patients were considered stable. It considers ten variables and yields scores of 0-100. A high score is associated with a greater likelihood of being able to live at home with a good degree of independence after discharge from hospital. Subjects can be divided into five levels of dependence: total (scores of 0-24), severe (scores of 25-49), moderate (scores of 50-74), mild (scores of 75-90) and minimal impairment (scores of 91-100). In order to avoid ceiling effects, we excluded any patients with a score lower than 15 , so as to exclude most patients with the highest degree of physical impairment, in whom it might be more difficult to detect any potential effects of anticholinergic drugs on the basic activities of daily living.

\subsection{Statistical Analysis}

Analysis of variance was used to study the relationship between drug-related anticholinergic burden and cognitive performance as assessed using the SBT or BI, both in univariate and multivariate models. Multivariate analyses of the association between SBT and anticholinergic drug use were adjusted for the known risk factors such as age, sex, education, and history of stroke or transient ischaemic attack (TIA) and number of non-anticholinergic drugs as possible confounders, while analyses for the association between 
anticholinergic drug use and BI were adjusted for age, sex and CIRS severity index. In view of non-normality in SBT and BI scores, the models were also evaluated using logistic regressions with SBT and BI cut point intervals as response variables, obtaining the significance reported in Tables 2, 3, 4, 5. The Pearson correlation coefficient of the univariate model was used to study the association between the change in the BI score and change in ACB or ARS scores. Analyses were done with JMP Pro 9 (SAS Institute Inc., Cary, NC, USA).

\section{Results}

3.1 Anticholinergic Drug Exposure According to the Anticholinergic Cognitive Burden (ACB) Scale and Anticholinergic Risk Scale (ARS)

Sixty-six internal medicine and geriatric wards participated in the REPOSI study and recruited 1,380 patients. Of these, 1,232 had SBT scores between 0 and 24 and were included. According to the ACB scale and ARS, respectively, 724
$(58.8 \%)$ and $112(9.1 \%)$ patients received at least one anticholinergic drug. Table 6 summarizes their sociodemographic characteristics. The most commonly prescribed anticholinergic medications (Table 7) are those with possible anticholinergic effects but no known clinically relevant cognitive effects according to the ACB scale (score of 1) or with a low risk of anticholinergic adverse effects according to the ARS (score of 1). Multivariate analysis, adjusted for age, sex and CIRS severity index found no differences in length of hospital stay between anticholinergic non-users (mean 10.6 days; $95 \%$ CI 9.9-11.3) and anticholinergic drug users (mean 10.9 days; $95 \%$ CI $10.3-11.5 ; p=0.56$ ) according to the ACB scale. Similar results were found with the ARS between anticholinergic non-users (mean 10.8 days; $95 \%$ CI 10.3-11.2) and anticholinergic drug users (mean 10.7 days; $95 \%$ CI 9.2-12.2; $p=0.92$ ). No association between the length of stay and the anticholinergic burden was found when the total score of the ACB scale or ARS was used as a continuous variable (slope $=0.04, p=0.86$, for ACB scale; slope $=0.10$, $p=0.82$, for ARS).

Table 2 Association between Short Blessed Test (SBT) ${ }^{\mathrm{a}}$ and anticholinergic drug use in patients with SBT scores of 0-24

\begin{tabular}{|c|c|c|c|c|c|c|}
\hline \multirow[t]{2}{*}{ Analysis } & \multicolumn{3}{|c|}{ Any anticholinergic drug according to ACB scale } & \multicolumn{3}{|c|}{ Any anticholinergic drug according to ARS } \\
\hline & Use $(n=724)$ & Non-use $(n=508)$ & $p$ value & Use $(n=112)$ & Non-use $(n=1,120)$ & $p$ value \\
\hline \multicolumn{7}{|l|}{ Univariate analysis } \\
\hline Median (IQR) & $8(3-14)$ & $6(2-12)$ & & $10(4-14)$ & $8(2-13)$ & \\
\hline Mean $(95 \%$ CI $)$ & $8.9(8.4-9.4)$ & $7.8(7.2-8.4)$ & 0.006 & $9.7(8.4-10.9)$ & $8.3(7.9-8.7)$ & 0.04 \\
\hline 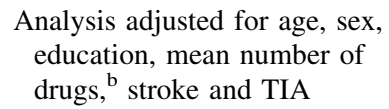 & $9.2(8.6-9.9)$ & $8.5(7.8-9.2)$ & 0.05 & $9.9(8.7-11.2)$ & $8.8(8.2-9.4)$ & 0.07 \\
\hline
\end{tabular}

Table 3 Dose-response relationship in models adjusted for age, education, mean number of drugs, ${ }^{\text {a }}$ stroke and transient ischaemic attack

\begin{tabular}{|c|c|c|c|c|c|}
\hline \multicolumn{3}{|l|}{ ACB scale and SBT scores } & \multicolumn{3}{|l|}{ ARS and SBT scores } \\
\hline Sum of ACB scale scores & Patients (n) & $\mathrm{SBT}^{\mathrm{b}}[$ mean $(95 \% \mathrm{CI})]$ & Sum of ARS scores & Patients (n) & $\mathrm{SBT}^{\mathrm{b}}[$ mean $(95 \% \mathrm{CI})]$ \\
\hline 0 & 508 & $8.5(7.8-9.2)$ & 0 & 1,120 & $8.8(8.2-9.4)$ \\
\hline 1 & 407 & $9.1(8.3-9.9)$ & 1 & 73 & $10.3(8.8-11.8)$ \\
\hline 2 & 200 & $9.3(8.3-10.2)$ & 2 & 23 & $8.7(6.1-11.3)$ \\
\hline 3 & 80 & $9.5(7.9-11.0)$ & $\geq 3$ & 16 & $9.8(6.6-13.1)$ \\
\hline 4 & 23 & $9.4(6.8-12.1)$ & & & \\
\hline$\geq 5$ & 14 & $10.6(7.3-13.9)$ & & & \\
\hline
\end{tabular}

$A C B$ Anticholinergic Cognitive Burden, ARS Anticholinergic Risk Scale, CI confidence interval, SBT Short Blessed Test

${ }^{\text {a }}$ Excluding anticholinergic drugs

b Higher scores indicate worse cognition 
Table 4 Association between Barthel Index ${ }^{a}$ during hospital stay and anticholinergic drug use in patients with Short Blessed Test (SBT) scores of $0-24$

\begin{tabular}{|c|c|c|c|c|c|c|}
\hline \multirow[t]{2}{*}{ Analysis } & \multicolumn{3}{|c|}{ Any anticholinergic drug according to ACB scale } & \multicolumn{3}{|c|}{ Any anticholinergic drug according to ARS } \\
\hline & Use $(n=681)$ & Non-use $(n=487)$ & $p$ value & Use $(n=102)$ & Non-use $(n=1,066)$ & $p$ value \\
\hline \multicolumn{7}{|l|}{ Univariate analysis } \\
\hline Median (IQR) & $92(73-100)$ & $98(85-100)$ & & $85(62.5-100)$ & $95(80-100)$ & \\
\hline Mean $(95 \% \mathrm{CI})$ & $82.4(80.8-84.0)$ & $87.8(85.9-89.7)$ & $<0.0001$ & $77.5(73.3-81.6)$ & $85.4(84.1-86.7)$ & 0.0004 \\
\hline $\begin{array}{l}\text { Analysis adjusted for age, } \\
\text { sex and CIRS severity index }\end{array}$ & $83.5(81.9-85.0)$ & $86.3(84.4-88.1)$ & 0.03 & $79.0(74.9-83.0)$ & $85.2(84.0-86.4)$ & 0.006 \\
\hline
\end{tabular}

$A C B$ Anticholinergic Cognitive Burden, ARS Anticholinergic Risk Scale, CI confidence interval, CIRS Cumulative Illness Rating Scale, IQR interquartile range

${ }^{\text {a }}$ Higher scores indicate less impairment

Table 5 Dose-response relationship in models adjusted for age, education, mean number of drugs, stroke and transient ischaemic attack

$A C B$ Anticholinergic Cognitive Burden, ARS Anticholinergic Risk Scale, $B I$ Barthel Index, $C I$ confidence interval

${ }^{\mathrm{a}}$ Higher scores indicate less impairment

\begin{tabular}{|c|c|c|c|c|c|}
\hline \multicolumn{3}{|c|}{ ACB scale and BI scores } & \multicolumn{3}{|c|}{ ARS and BI scores } \\
\hline $\begin{array}{l}\text { Sum of ACB } \\
\text { scale scores }\end{array}$ & $\begin{array}{l}\text { Patients } \\
\text { (n) }\end{array}$ & $\begin{array}{l}\mathrm{BI}^{\mathrm{a}} \\
{[\text { mean }(95 \% \mathrm{CI})]}\end{array}$ & $\begin{array}{l}\text { Sum of ARS } \\
\text { scores }\end{array}$ & $\begin{array}{l}\text { Patients } \\
\text { (n) }\end{array}$ & $\begin{array}{l}\mathrm{BI}^{\mathrm{a}} \\
{[\text { mean }(95 \% \mathrm{CI})]}\end{array}$ \\
\hline 0 & 487 & $86.3(84.4-88.1)$ & 0 & 1,066 & $85.2(84.0-86.4)$ \\
\hline 1 & 382 & $83.0(81.0-85.1)$ & 1 & 67 & $81.4(76.4-86.4)$ \\
\hline 2 & 190 & $83.9(80.9-86.8)$ & 2 & 20 & $77.8(68.7-86.8)$ \\
\hline 3 & 73 & 85.7 (80.9-90.4) & $\geq 3$ & 15 & 71.5 (61.1-81.9) \\
\hline 4 & 22 & 85.5 (76.9-94.2) & & & \\
\hline$\geq 5$ & 14 & $77.3(66.4-88.2)$ & & & \\
\hline
\end{tabular}

Table 6 Sociodemographic characteristics of patients with Short Blessed Test scores of 0-24 according to anticholinergic drug use at admission

\begin{tabular}{|c|c|c|c|c|c|c|}
\hline \multirow[t]{2}{*}{ Characteristic } & \multicolumn{3}{|l|}{ ACB scale } & \multicolumn{3}{|l|}{ ARS } \\
\hline & Use & Non-use & $p$ value & Use & Non-use & $p$ value \\
\hline Number of patients & 724 & 508 & & 112 & 1,120 & \\
\hline Age (years; mean $\pm \mathrm{SD}$ ) & $79.0 \pm 7.3$ & $78.0 \pm 7.0$ & 0.02 & $79.1 \pm 7.2$ & $78.5 \pm 7.2$ & 0.42 \\
\hline Female $(\%)$ & 50.3 & 47.2 & 0.29 & 58.0 & 48.1 & 0.05 \\
\hline Number of drugs ${ }^{\mathrm{a}}$ (mean $\pm \mathrm{SD}$ ) & $4.9 \pm 2.4$ & $3.7 \pm 2.2$ & $<0.0001$ & $5.5 \pm 2.7$ & $5.2 \pm 2.8$ & 0.33 \\
\hline Education (years; mean \pm SD) & $7.1 \pm 4.1$ & $7.4 \pm 4.2$ & 0.22 & $7.3 \pm 4.2$ & $7.2 \pm 4.1$ & 0.85 \\
\hline \multicolumn{7}{|l|}{ CIRS } \\
\hline Diagnosis (n; mean \pm SD) & $6.3 \pm 2.8$ & $4.9 \pm 2.5$ & $<0.0001$ & $6.5 \pm 3.0$ & $5.6 \pm 2.7$ & 0.006 \\
\hline Severity index score (mean \pm SD) & $1.7 \pm 0.3$ & $1.5 \pm 0.3$ & $<0.0001$ & $1.7 \pm 0.3$ & $1.6 \pm 0.3$ & 0.02 \\
\hline Comorbidity index score (mean $\pm \mathrm{SD}$ ) & $3.2 \pm 1.7$ & $2.4 \pm 1.6$ & $<0.0001$ & $3.4 \pm 1.8$ & $2.8 \pm 1.7$ & 0.002 \\
\hline
\end{tabular}

$A C B$ Anticholinergic Cognitive Burden, ARS Anticholinergic Risk Scale, CIRS Cumulative Illness Rating Scale

${ }^{a}$ Excluding anticholinergic drugs

\subsection{Relationship between Anticholinergic Burden and Short Blessed Test (SBT)}

\subsubsection{ACB Scale and SBT}

The SBT was available for 1,232 patients. According to the ACB scale, 724 patients were treated with at least one anticholinergic drug (Tables 2 and 3). In the univariate model, the mean SBT score of patients treated with anticholinergic drugs $(8.9 ; 95 \%$ CI 8.4-9.4) was about one point higher than that of patients without any anticholinergic medications $(7.8 ; 95 \%$ CI 7.2-8.4; $p=0.006)$. The difference was similar after adjustment for age, sex, education, and history of stroke or TIA. The mean SBT score of patients treated with anticholinergic drugs (9.2; $95 \%$ CI 8.6-9.9) was significantly higher than the score $(8.5 ; 95 \%$ CI 7.8-9.2) for patients without anticholinergic drugs $(p=0.05)$. A dose-response relationship was 
Table 7 Anticholinergic medications most commonly prescribed according to the Anticholinergic Cognitive Burden (ACB) Scale and Anticholinergic Risk Scale (ARS)

\begin{tabular}{llll}
\hline Scale & Drug & Score & $\begin{array}{l}\text { Anticholinergic } \\
\text { users }(n)\end{array}$ \\
\hline ACB scale & Furosemide & 1 & 439 \\
& Warfarin & 1 & 143 \\
& Digoxin & 1 & 98 \\
& Prednisone & 1 & 63 \\
& Isosorbide & 1 & 52 \\
& Atenolol & 1 & 44 \\
Metoprolol & 1 & 37 \\
& Triamterene & 1 & 26 \\
& Alprazolam & 1 & 25 \\
& Nifedipine & 1 & 24 \\
& Carbidopa - levodopa & 1 & 27 \\
& Ranitidine & 1 & 26 \\
& Paroxetine & 1 & 17 \\
& Quetiapine & 1 & 9 \\
Haloperidol & 1 & 8 \\
Pramipexole & 1 & 8 \\
Amitriptyline & 3 & 7 \\
& Pseudoephedrine & 2 & 4 \\
Cetirizine & 2 & 3 \\
Olanzapine & 2 & 2 \\
\hline & & &
\end{tabular}

observed between higher total ACB score and cognitive impairment; patients who scored 1 had a 0.6-point decline in SBT scores. A plateau effect was found in patients who scored 2-4, with 0.8-, 0.9- and 1.0-point declines, respectively; patients who scored 5 or more had about a 2.1-point greater decline than those not taking anticholinergic drugs. Results of the univariate and multivariate analyses were similar after inclusion of 13 demented patients (previously excluded for the diagnosis of dementia or treatment with anticholinesterase agents or memantine). In the multivariate model, the mean SBT score for patients treated with anticholinergic drugs $(9.4 ; 95 \%$ CI 8.7-10.1) was higher than the mean score $(8.7 ; 95 \%$ CI 8.0-9.4) for patients without anticholinergic drugs $(p=0.07)$, although the difference was only marginally significant. The doseresponse relationship was maintained.

\subsubsection{ARS and SBT}

The ARS identified 112 patients receiving at least one anticholinergic drug. In the univariate model, the mean SBT score of patients treated with anticholinergic drugs $(9.7 ; 95 \%$ CI $8.4-10.9)$ was 1.4 points higher than that of patients receiving no anticholinergic medication $(8.3 ; 95$ $\%$ CI $7.9-8.7 ; p=0.04)$. In the multivariate model, after adjustment for age, sex education, stroke and TIA, the difference was only marginally significant (Tables 2 and 3) and there was no dose-response relationship between higher total ARS score and cognitive decline. Results were similar after inclusion of 13 demented patients; in the multivariate analysis, the mean SBT score for patients treated with anticholinergic drugs $(9.8 ; 95 \%$ CI $8.5-11.0)$ was higher than the mean score $(8.4 ; 95 \%$ CI $8.0-8.8)$ for patients without anticholinergic drugs $(p=0.05)$.

The results of univariate and multivariate analyses after exclusion of patients with a diagnosis of delirium $(n=8)$ at hospital admission did not differ for both the ACB scale and ARS.

\subsection{Relationship between Anticholinergic Burden and Barthel Index (BI)}

\subsubsection{ACB Scale and BI}

Out of the 1,245 patients in the analysis of the association between drug-related anticholinergic burden and SBT, the BI was available for 1,167 at admission. In the univariate analysis, the use of anticholinergic drugs was associated with greater impairment in basic activities of daily living (Tables 4 and 5). The mean BI score of patients treated with anticholinergic drugs $(82.4 ; 95 \%$ CI 80.8-84.0) was about 5 points lower than that of patients receiving no anticholinergic medication (87.8; $95 \%$ CI 85.9-89.7; $p<0.0001)$. The independent association between cumulative anticholinergic exposure and poorer BI rating was maintained after adjustment for age, sex and CIRS severity index, the mean BI score for patients treated with anticholinergic drugs being 83.5 (95\% CI 81.9-85.0) as opposed to 86.3 (95\% CI 84.4-88.1; $p=0.03$ ) for those not treated with these drugs. No dose-response relationship was been observed.

BI scores were available for 666 patients at the 3-month follow-up; the mean BI score adjusted for age, sex and CIRS severity index was 82.8 (95\% CI 80.7-84.9) in anticholinergic drug users and 86.7 (95\% CI 84.2-89.2) in non-users $(p=0.02)$. Analysis of the dose-response relationship confirmed that patients who scored 4 or more had a higher degree of impairment (mean BI score 80.7; $95 \%$ CI 72.5-89.0; $p<0.0001)$.

\subsubsection{ARS and BI}

The ARS identified 102 patients with at least one anticholinergic drug and a BI rating. The mean BI score at admission was significantly lower for patients using anticholinergic medications in both univariate and multivariate models. After adjustment for age, sex and CIRS severity index, the mean BI scores were 79.0 (95\% CI 74.9-83.0) 
in anticholinergic drug users and 85.2 (95\% CI 84.0-86.4) in non-users $(p=0.006)$ (Tables 4 and 5). A doseresponse relationship was observed between higher total ARS score and ability in basic activities of daily living. The association was confirmed at follow-up. In the multivariate model, the mean BI score was 76.9 (95\% CI 71.8-82.1) in anticholinergic drug users and 85.2 (95\% CI 83.5-86.9) in non-users $(p=0.003)$. A dose-response relationship was also observed, the mean BI score of patients who scored 3 or more being 68.7 (95\% CI 56.4-81.0; $p<0.0001)$.

No correlation between change in anticholinergic burden and change in BI score at 3 months after hospital admission was found (correlation $=0.004, p=0.91$, for ACB scale; correlation $=-0.06, p=0.15$, for ARS).

\subsection{Relationship between ACB Scale and ARS}

Our results suggest that the ARS could be more specific but less selective than the ACB scale in identifying patients with a higher degree of cognitive impairment (Table 8). Patients identified as not receiving anticholinergic drugs in both scales were those with better cognitive performance and ability in basic activities of daily living, while patients identified only in the ARS had higher levels of cognitive and physical impairment, although anticholinergic burden was relatively low; one patient scored 3 , four scored 2 and 16 scored 1. Neither the ACB scale nor the ARS were associated with longer hospital stay.

\section{Discussion}

Consistent with our primary hypothesis, the cumulative effects of anticholinergic drugs, as assessed by both the ACB scale and ARS, were associated with cognitive and functional impairment in a sample of elderly patients. The ACB scale was more selective in capturing medications with modest or in vitro antimuscarinic activity likely to affect cognitive and physical performance, while the ARS was more specific in capturing medication associated with a higher degree of cognitive and functional of impairment. No study has previously compared these two anticholinergic scales. A recent, large, population-based study on anticholinergic drugs using the ACB scale found an increasing risk for cognitive decline (as measured by the Mini-Mental State Examination) and mortality over 2 years in patients with normal or mildly impaired cognition [42]. It has also been postulated that patients with Alzheimer's disease (AD) may be at particular risk of cognitive deterioration with the use of medications with anticholinergic effects, because of the marked reduction in the functioning of central cholinergic pathways [19, 43]. However, a recent study did not confirm this, concluding that in patients with $\mathrm{AD}$, medications with anticholinergic effect according to the ACB scale did not affect deterioration in cognition over the subsequent 18 months, thus not supporting a continuous effect of these medications on patients with $\mathrm{AD}$ [44].

The ARS score has also been associated with the risk of central and peripheral anticholinergic adverse effects in elderly patients. However, there are some differences between the ACB scale and ARS: their lists of medications with anticholinergic activity include a different number of drugs and score some drugs differently. Furosemide, warfarin and digoxin are not included in the ARS; paroxetine and quetiapine are classified differently; they are considered drugs with definite, clinically relevant anticholinergic effects in the ACB scale (score of 3), while the ARS assigns them a low risk of anticholinergic effects (score of 1).

In the present study, both the ACB scale and ARS identified patients with greater cognitive impairment among those with no clinical diagnosis of dementia. According to the ACB scale, the most prescribed anticholinergic drugs are those with only serum anticholinergic activity or in vitro affinity for muscarinic receptors with no clinically relevant cognitive effects. This list includes furosemide, warfarin, digoxin, prednisone and isosorbide. However, the
Table 8 Patients treated with anticholinergic drugs according to Anticholinergic Cognitive Burden (ACB) Scale and Anticholinergic Risk Scale (ARS)

$B I$ Barthel Index, $C I$ confidence interval, SBT Short Blessed Test

\begin{tabular}{cllll}
\hline \multicolumn{5}{c}{ Anticholinergic use } \\
\cline { 2 - 5 } & $\begin{array}{l}\text { Non-use in ACB } \\
\text { scale and ARS }\end{array}$ & $\begin{array}{l}\text { Use only in } \\
\text { ACB scale }\end{array}$ & $\begin{array}{l}\text { Use in ACB } \\
\text { scale and ARS }\end{array}$ & $\begin{array}{l}\text { Use only } \\
\text { in ARS }\end{array}$ \\
\hline Association with SBT & & & & \\
Number of patients & 487 & 633 & 91 & 21 \\
SBT score [mean & $7.7(7.1-8.2)$ & $8.9(8.3-9.4)$ & $9.2(7.8-10.7)$ & $11.6(7.8-15.5)$ \\
$\quad(95 \%$ CI)] & & & & \\
Association with BI & & 631 & 91 & 21 \\
Number of patients & 484 & $78.8(76.6-80.1)$ & $73.0(66.7-79.4)$ & $62.8(47.4-78.1)$ \\
BI score [mean & $85.8(83.7-88.0)$ & & \\
$\quad(95 \%$ CI)] & & & & \\
\hline
\end{tabular}


cumulative anticholinergic effects of these drugs have been associated with greater cognitive impairment and a doseresponse relationship; according to our results patients who scored 5 or more had a 2-point cognitive decline according to the SBT. The clinical significance of a 2-point difference is difficult to clarify because the rate of decline is generally determined by the severity of dementia; the less severe the dementia, the slower the rate of decline. The annual rate of decline on the SBT is about 3.8 points in patients with probable $\mathrm{AD}$ [48], and a 2-point difference could be compare to a 6-month decline in patients with probable AD. In any case, people receiving drugs with a combined ACB scale score of 5 were found to have higher cognitive impairment than those who took none of these drugs. This would be enough to impair concentration and driving ability and, additionally, in someone already experiencing cognitive impairment, worsen memory problems, personality changes or depression [49].

For many drugs with important clinical indications, such as warfarin or digoxin, it is hard to suggest non-anticholinergic alternative drugs and it may be difficult to reduce the anticholinergic burden. However, when there are alternative drug choices, every attempt should be made to avoid increasing a patient's anticholinergic burden. An association was also observed between drug-related anticholinergic burden and impairment in basic activities of daily living, although no dose-response relationship was found, but patients who scored 5 or more had greater physical impairment. In the ARS, no dose-response relationship with cognitive impairment was found, while there was a clear relationship with the BI scale, suggesting that the ARS should be the preferred scale for identifying patients at higher risk of physical impairment.

Delirium is an acute cognitive failure and its relationship with anticholinergic medication has been debated [50, 51]. In order to avoid the cognitive impairment related to delirium, the SBT was conducted within 2 days of hospital admission or after the patient stabilized. Analysis repeated after inclusion of patients with a diagnosis of delirium gave similar results for the ACB scale and ARS.

A limitation of this study is the lack of information on the SBT at follow-up, because changes in a patient's drug regimen may have far-reaching effects on their anticholinergic burden. To date, no clinical trial has evaluated the cognitive outcome of reducing anticholinergic burden, and the lack of information on the SBT at follow-up excluded the possibility of assessing the relationship between longitudinal cumulative anticholinergic exposure and cognitive performance. We also did not examine the effects of different doses. Another limitation of the study is that the association between anticholinergic burden and cognitive or functional outcome was a hypothesis: sicker patients (with more cognitive or functional impairment) could be more likely to be prescribed an anticholinergic medication. However, biological, rational [19] and other observational studies [22-25, 42] support the hypothesis that the cumulative effects of anticholinergic drugs are associated with cognitive and functional impairment. For the association between anticholinergic drug use and cognitive impairment, we adjusted analyses for possible confounders such as age, sex, education, and history of stroke or TIA and number of non-anticholinergic drugs, and, similarly, for the association between anticholinergic drug use and physical impairment, we adjusted for age, sex and CIRS severity index, thus, limiting the potential bias of confounding by indication. Another limitation is that the REPOSI study was not designed to specifically collect the diagnosis of dementia; we focused on patients with SBT scores of 0-24 in order to avoid the ceiling effects of this scale, thus, excluding the majority of demented patients. The distribution of patients with a diagnosis of dementia showed that $53 \%$ had SBT scores between 25 and 28. However, some patients with a diagnosis of dementia may have been included.

\section{Conclusions}

Prescribers need to be vigilant for adverse anticholinergic effects, particularly in older patients. The effects of anticholinergic drugs on cognitive and physical performance might be due to the cumulative effect of multiple medications with modest antimuscarinic activity. Different sets of explicit criteria for potentially inappropriate medication, such as the Beers [45] or Screening Tool to Alert Doctors to Right Treatment/Screening Tool of Older Person's Prescriptions (START/STOPP) criteria [46], have been developed to reduce the use of drugs that have no clear evidence-based indication or that involve a substantial risk of adverse effects in elderly patients [47]. Evaluation of anticholinergic burden should be considered an additional important strategy for optimizing polypharmacy, and the incorporation of these scales into computer-assisted order entry systems would facilitate evaluation of the anticholinergic burden in daily practice. According to the results of this study, the ACB scale might help rapidly identify drugs potentially associated with cognitive impairment with a dose-response pattern, while the ARS seems better used for identifying patients with impairment in activities of daily living.

Acknowledgments The REPOSI study is a network of Italian internal medicine hospital wards that, voluntarily and without any financial support, agreed to participate in data collection during the 4 index weeks. All authors had full access to all data and take responsibility for its integrity and the accuracy of the data analysis. All authors reviewed the manuscript and contributed to the 
interpretation of results. The authors have no conflict of interest. We are grateful to Judith Baggott for editorial assistance.

\section{References}

1. Veehof LJG, Stewart RE, Haaijer-Raskamp FM, et al. The development of polypharmacy. A longitudinal study. Fam Pract. 2000;17:261-7.

2. Gurwitz JH. Polypharmacy. A new paradigm for quality drug therapy in the elderly? Arch Intern Med. 2004;164:1957-9.

3. Hajjar ER, Cafiero AC, Hanlon JT. Polypharmacy in elderly patients. Am J Geriatr Pharmacother. 2007;5:345-51.

4. Hilmer SN, Gnjidic D. The effects of polypharmacy in older adults. Clin Pharmacol Ther. 2009;85:86-98.

5. Steinman MA, Landefeld CS, Rosenthal GE, et al. Polypharmacy and prescribing quality in older people. J Am Geriatr Soc. 2006; 54:1516-23.

6. Koh Y, Kutty FBM, Li SC. Drug-related problems in hospitalized patients on polypharmacy: the influence of age and gender. Ther Clin Risk Manag. 2005;1:39-48.

7. Lund $\mathrm{BC}$, Camahan RM, Egge JA, et al. Inappropriate prescribing predicts adverse drug events in older adults. Ann Pharmacother. 2010. doi:10.1345/aph.1M657.

8. Nobili A, Garattini S, Mannucci PM. Multiple diseases and polypharmacy in the elderly: challenges for the internist of the third millennium. J Comorbidity. 2011;1:28-44.

9. Flacker JM, Cummings V, Mach JR Jr, et al. The association of serum anticholinergic activity with delirium in elderly medical patients. Am J Geriatr Psychiatry. 1998;6(1):31-41.

10. Tune LE. Anticholinergic effects of medication in elderly patients. J Clin Psychiatry. 2001;62(suppl 21):11-4.

11. Hanlon JT, Schamder KE, Boult C, et al. Use of inappropriate prescription drugs by older people. J Am Geriatr Soc. 2002;50:26-34.

12. Boustani M, Hall KS, Lane KA, et al. The association between cognition and histamine-2 receptor antagonists in African Americans. J Am Geriatr Soc. 2007;55:1248-53.

13. Castelino RL, Bajorek BV, Chen TF. Targeting suboptimal prescribing in the elderly: a review of the impact of pharmacy services. Ann Pharmacother. 2009;43:1096-106.

14. Campbell NL, Boustani MA, Lane KA, et al. Use of anticholinergics and the risk of cognitive impairment in an African American population. Neurology. 2010;75:152-9.

15. Carriere I, Fourrier-Reglat A, Dartigues JF, et al. Drugs with anticholinergic properties, cognitive decline, dementia in an elderly general population-the 3-City study. Arch Intern Med. 2009;169:1317-24.

16. Mulsant BH, Pollock BG, Kirshner M, Shen C, Dodge H, Ganguli M. Serum anticholinergic activity in a community-based sample of older adults: relationship with cognitive performance. Arch Gen Psychiatry. 2003;60(2):198-203.

17. Chew ML, Mulsant BH, Pollock BG. Serum anticholinergic activity and cognition in patients with moderate-to-severe dementia. Am J Geriatr Psychiatry. 2005;13(6):535-8.

18. Lechevallier-Michel N, Molimard M, Dartigues JF, et al. Drugs with anticholinergic properties and cognitive performance in the elderly: results from the PAQUID study. Br J Clin Pharmacol. 2005;59(2):143-51.

19. Roe CM, Anderson MJ, Spivack B. Use of anticholinergic medications by older adults with dementia. J Am Geriatr Soc. 2002;50(5):836-42.

20. Han L, McCusker J, Cole M, et al. Use of medications with anticholinergic effect predicts clinical severity of delirium symptoms in older medical inpatients. Arch Intern Med. 2001;161(8):1099-105.
21. Moore AR, O'Keeffe ST. Drug-induced cognitive impairment in the elderly. Drugs Aging. 1999;15(1):15-28.

22. Aizenberg D, Sigler M, Weizman A, et al. Anticholinergic burden and the risk of falls among elderly psychiatric inpatients: a 4-year case-control study. Int Psychogeriatr. 2002;14(3):307-10.

23. Ancelin ML, Artero S, Portet F, et al. Non-degenerative mild cognitive impairment in elderly people and use of anticholinergic drugs: longitudinal cohort study. BMJ. 2006;332(7539):455-9.

24. Landi F, Russo A, Liperoti R, et al. Anticholinergic drugs and physical function among frail elderly population. Clin Pharmacol Ther. 2007;81(2):235-41.

25. Low LF, Anstey KJ, Sachdev P. Use of medication with anticholinergic properties and cognitive function in a young-old community sample. Int Geriatr Psychiaty. 2009;24:578-84.

26. Atkin PA, Veitch PC, Veitch EM, et al. The epidemiology of serious adverse drug reactions among the elderly. Drugs Aging. 1999; 14(2):141-52.

27. Hutchinson TA, Flegel KM, Kramer MS, et al. Frequency, severity and risk factors for adverse drug reactions in adult outpatients: a prospective study. J Chronic Dis. 1986;39(7):533-42.

28. Rudolph JL, Salow MJ, Angelini MC, et al. The anticholinergic risk scale and anticholinergic adverse effects in older person. Arch Intern Med. 2008;168(5):508-13.

29. Buostani M, et al. Impact of anticholinergics on the aging brain: a review and practical application. Aging Health. 2008;4(3):311-20.

30. Nobili A, Marengoni A, Tettamanti M, et al. Association between clusters of diseases and polypharmacy in hospitalized elderly patients: results from the REPOSI study. Eur J Intern Med. 2011;22(6):597-602.

31. Marengoni A, Corrao S, Nobili A, et al. In-hospital death according to dementia diagnosis in acutely ill elderly patients: the REPOSI study. Int J Geriatr Psychiatry. 2011;26(9):930-6.

32. Pasina L, Nobili A, Tettamanti M, et al. Prevalence and appropriateness of drug prescriptions for peptic ulcer and gastroesophageal reflux disease in a cohort of hospitalized elderly. Eur J Intern Med. 2011;22(2):205-10.

33. Nobili A, Licata G, Salerno F, et al. Polypharmacy, length of hospital stay, and in-hospital mortality among elderly patients in internal medicine wards. The REPOSI study. Eur J Clin Pharmacol. 2011;67(5):507-19.

34. Marcucci M, Iorio A, Nobili A, et al. Factors affecting adherence to guidelines for antithrombotic therapy in elderly patients with atrial fibrillation admitted to internal medicine wards. Eur J Intern Med. 2010;21(6):516-23.

35. Marengoni A, Corrao S, Nobili A, et al. The participating units and co-authors are listed in the Appendix. In-hospital death according to dementia diagnosis in acutely ill elderly patients: the REPOSI study. Int J Geriatr Psychiatry. 2010.

36. Parmelee PA, Thuras PD, Katz IR, Lawton MP. Validation of the Cumulative Illness Rating Scale in a geriatric residential population. J Am Geriatr Soc. 1995;43(2):130-7.

37. Shah S, Vanclay F, Cooper B. Improving the sensitivity of the Barthel Index for stroke rehabilitation. J Clin Epidemiol. 1989;42(8):703-9.

38. Katzman R, Brown T, Fuld P, et al. Validation of a short orientation-memory-concentration test of cognitive impairment. Am J Psychiatry. 1983;140(6):734-9.

39. Hickie C, Snowdon J. Depression scales for the elderly: GDS, Gilleard, Zung. Clin Gerontol. 1987;6(3):51-3.

40. Carnahan RM, et al. The anticholinergic drug scale as a measure of drug-related anticholinergic burden: association with serum anticholinergic activity. J Clin Pharmacol. 2006;46:1481-6.

41. Morris JC, Heyman A, Mohs RC, et al. The Consortium to Establish a Registry for Alzheimer's Disease (CERAD). Part I. Clinical and neuropsychological assessment of Alzheimer's disease. Neurology. 1989;39(9):1159-65. 
42. Fox C, Richardson K, Maidment ID, et al. Anticholinergic medication use and cognitive impairment in the older population: the medical research council cognitive function and ageing study. J Am Geriatr Soc. 2011;59(8):1477-83.

43. Sunderland T, Tariot PN, Cohen RM, et al. Anticholinergic sensitivity in patients with dementia of the Alzheimer's type and age-matched controls: a dose response study. Arch Gen Psychiatry. 1987;44:418-26.

44. Fox C, Livingstone G, Maidment I, et al. The impact of anticholinergic burden in Alzheimer's dementia-the laser AD study. Age Ageing. 2011;40:730-5.

45. Fick DM, Cooper JW, Wade WE, et al. Updating the Beers criteria for potentially inappropriate medication use in older adults: results of a US consensus panel of experts. Arch Intern Med. 2003;163:2716-24.

46. Gallagher P, Ryan C, Byrne S, et al. STOPP (Screening Tool of Older Persons' Prescriptions) and START (Screening Tool to Alert Doctors to Right Treatment): consensus validation. Int $\mathbf{J}$ Clin Pharmacol Ther. 2008;46(2):72-83.
47. Chang CB, Chan DC. Comparison of published explicit criteria for potentially inappropriate medications in older adults. Drugs Aging. 2010;27(12):947-57.

48. Morris JC, Edland S, Clark C, et al. The Consortium to Establish a Registry for Alzheimer's Disease (CERAD). Part V. Rates of cognitive change in the longitudinal assessment of probable Alzheimer's disease. Neurology. 1993;43:2457-65.

49. Torijesen I. Anticholinergic effects of common drugs are associated with increased mortality in over 65s. BMJ. 2011;342: d3514.

50. Campbell N, Perkins A, Hui S, Khan B, Boustani M. Association between prescribing of anticholinergic medications and incident delirium: a cohort study. J Am Geriatr Soc. 2011;59(Suppl 2):S277-81. doi:10.1111/j.1532-5415.2011.03676.x.

51. Flacker JM, Wei JY. Endogenous anticholinergic substances may exist during acute illness in elderly medical patients. J Gerontol A Biol Sci Med Sci. 2001;56(6):M353-5. 